\title{
Erratum to: Hepatoprotective Effect of Arctium lappa Root Extract on Cadmium Toxicity in Adult Wistar Rats
}

\author{
Fabricia de Souza Predes • Maria Aparecida da Silva Diamante • Mary Ann Foglio • \\ Camila de Andrade Camargo • Hiroshi Aoyama • Silvio Cesar Miranda • Bread Cruz • \\ Maria Cristina Cintra Gomes Marcondes • Heidi Dolder
}

Published online: 24 July 2014

(C) Springer Science+Business Media New York 2014

Erratum to: Biol Trace Elem Res (2014) 160:250-257

\section{DOI 10.1007/s12011-014-0040-6}

Due to an oversight, Dr. Camila de Andrade Camargo's name was listed incorrectly (as Camila Almeida Camargo) in the article Hepatoprotective Effect of Arctium lappa Root Extract on Cadmium Toxicity in Adult Wistar Rats, BTER, June 2014, DOI 10.1007/s12011-014-0040-6.

The online version of the original article can be found at http:// dx.doi.org/10.1007/s12011-014-0040-6.

F. de Souza Predes $(\bowtie)$

Departamento de Ciências Biológicas, Universidade Estadual do Paraná/Campus Paranaguá, Rua Comendador Correia Junior,

117-Centro, Paranaguá, Paraná 83203-560, Brazil

e-mail: fpredes@yahoo.com.br

M. A. da Silva Diamante - B. Cruz • M. C. C. Gomes Marcondes •

H. Dolder

Departamento de Biologia Estrutural e Funcional, Universidade

Estadual de Campinas, Campinas, São Paulo, Brazil

\section{A. Foglio}

Centro Pluridisciplinar de Pesquisas Químicas, Biológicas e

Agrárias, Universidade Estadual de Campinas, Campinas, São Paulo,

Brazil

C. de Andrade Camargo $\cdot$ H. Aoyama

Departamento de Bioquímica, Universidade Estadual de Campinas,

Campinas, São Paulo, Brazil

S. C. Miranda

Universidade Paulista, Campinas, São Paulo, Brazil 